\title{
Experiencias del liderazgo directivo en tiempos de covid-19.
}

Nieto-Rivas, Elier Abiud, Fermín Pérez, Félix Armando, Arieta Miranda, Yoly Marlene, Mondragón Silva, Doris Gerardina, Atanacio Cuaresmayo, Liliana y Meneses Jiménez, José Ángel.

Cita:

Nieto-Rivas, Elier Abiud, Fermín Pérez, Félix Armando, Arieta Miranda, Yoly Marlene, Mondragón Silva, Doris Gerardina, Atanacio Cuaresmayo, Liliana y Meneses Jiménez, José Ángel (2021). Experiencias del liderazgo directivo en tiempos de covid-19. South Florida Journal of Development, 2 (4), 6228-6245.

Dirección estable: https://www.aacademica.org/elier.nieto.rivas/2 ARK: https://n2t.net/ark:/13683/pDyd/vy3 


\title{
Experiencias del liderazgo directivo en tiempos de covid-19
}

\section{Experiences of managerial leadership in times of covid-19}

DOI: $10.46932 /$ sfjdv2n4-095

Received in: March 1st, 2021

Accepted in: May 30th, 2021

\author{
Nieto-Rivas, Elier Abiud \\ Magíster \\ Universidad César Vallejo \\ E-mail: enietori@ucvvirtual.edu.pe \\ Fermín Pérez, Félix Armando \\ Magíster \\ Universidad César Vallejo \\ E-mail: ffermin@ucvvirtual.edu.pe \\ Arieta Miranda, Yoly Marlene \\ Magíster \\ Universidad César Vallejo \\ E-mail: yolymarlene30@gmail.com \\ Mondragón Silva, Doris Gerardina \\ Magíster \\ Universidad César Vallejo \\ E-mail:dogmosil06@gmail.com \\ Atanacio Cuaresmayo, Liliana \\ Magíster \\ Universidad César Vallejo \\ E-mail: lunasli9152321@gmail.com \\ Meneses Jiménez, José Ángel \\ Doctor \\ Universidad César Vallejo \\ E-mail: jamenese@ ucvvirtual.edu.pe
}

\section{RESUMEN}

El liderazgo es un conjunto de tareas y actividades que realiza el líder de una organización con la finalidad de lograr metas establecidas, desarrollándose en diversos entornos como el social, laboral. En este sentido, el liderazgo de los directivos presenta gran interés en el actual contexto de trabajo remoto ocasionado por el Covid-19. En ese contexto, se plantea esta investigación que tiene como propósito aproximarse a las experiencias vividas por el personal directivo de las instituciones de educación de educación superior. El abordaje metodológico se enmarca dentro del enfoque cualitativo bajo un diseño de fenomenológico hermenéutico, en el que se usaron técnicas de observación participante y entrevistas a profundidad. Entre los principales resultados develados se encontró que el soporte emocional combinado con adecuados procesos de reflexión son elementos relevantes para influir positivamente en las instituciones educativas. 
Se llega a la conclusión que la dirección efectiva del liderazgo directivo depende en gran medida de la convergencia de las opiniones grupales y a delegación de funciones que conllevan a fortalecer el compromiso institucional.

Palabras clave: liderazgo directivo, trabajo pedagógico remoto, contexto de pandemia- Covid-19

\begin{abstract}
Leadership is a set of tasks and activities carried out by the leader of an organization in order to achieve established goals, developing in various environments such as social, work. In this sense, the leadership of managers is very interested in the current context of remote work caused by Covid-19. In this context, this research is proposed with the purpose of approaching the experiences lived by the directives of higher education institutions. The methodological approach is framed within the qualitative approach under a hermeneutical phenomenological design, in which participant observation techniques and in-depth interviews were used. Among the main results revealed, it was found that emotional support combined with adequate reflection processes are relevant elements to positively influence educational institutions. It is concluded that the effective direction of directive leadership depends to a great extent on the convergence of group opinions and the delegation of functions that lead to strengthening institutional commitment.
\end{abstract}

Keywords: executive leadership, remote pedagogical work, pandemic context, Covid-19

\title{
1 INTRODUCCIÓN
}

Betemi (2019), señala que el liderazgo es un conjunto de tareas y actividades que realiza el líder de una organización con la finalidad de lograr metas establecidas,desarrollándose en diversos entornos como el social, laboral, etc. Donde es importante resaltar que el líder ejerce influencia en los demás motivándolos a que realicen las funciones encomendadas de la mejor manera. La eficacia del liderazgo educativo está determinada por el resultado del aprendizaje del estudiante con la intención de mejorar la calidad educativa. Por todo lo antes mencionado, un buen líder debe congregar y lograr una convivencia armoniosa en la comunidad educativa (Amador, 2017).

En el Instituto de Educación Superior Pedagógico Público Víctor Raúl Haya de la Torre (IESPP VRHT), en el contexto de pandemia, se hace indispensable ejercer un liderazgo emergente que permita revertir la difícilsituación que atraviesa la organización educativa en la utilización de las Tecnologías de Información y Comunicación (TIC), para ejecutar el trabajo en la virtualidad, considerando además, que los directivos no estaban preparados para hacer frente a este desafío teniendo en cuenta que las TIC permiten interactuar con los estudiantes y lograr una comunicación sincrónica para desarrollar aprendizajes en todo ámbito educativo (Lescano, 2017).

Una de las causas de este problema es que el personal directivo docente,así como los estudiantes no estaban capacitados en el uso de herramientas tecnológicas, sin embargo, un director debe demostrar su liderazgo al enfrentarse a realidades desfavorables. Por otro lado, el recorte del presupuesto realizado 
por el Ministerio de Economía y Finanzas al Instituto de Educación Superior Pedagógico Público Víctor Raúl Haya de la Torre, ha afectado la carga horaria de los maestros trayendo consigo dificultades en el servicio brindado. Otra de las consecuencias, es la deserción de estudiantes por motivo de la falta de señal de internet o carencia de dispositivos electrónicos, una más lo constituye la excesiva recarga laboral de los maestros, debido a la reducción de los contratos, lo que ocasionó mayor estrés, aún. Estos retos debieron ser asumidos por el líder directivo rápidamente para construir diversas estrategias orientadas a mitigar la crisis ocasionada por la pandemia con la finalidad de continuar con el servicio educativo (Kochen, 2020).

Entre las consecuencias destaca que la pandemia ha permitido visualizar la brecha que existe en cuanto a lascondiciones tecnológicas debido al factor económico ya que tanto directivos, maestros o estudiantes no cuentan con la tecnología adecuada para la continuidad de las clases en el entorno virtual, por ello es que diversas aplicaciones como, por ejemplo, WhatsApp son considerados como una herramienta importante para realizar el acompañamiento pedagógico a losestudiantes (Bucheli \& Bastidas, 2020).

En el escenario objeto de estudio, se han propuesto el uso de diversas herramientas tecnológicas como: WhatsApp, Facebook, Google Meet, Google Classroom y la más reciente el Microsoft Teams. Sin embargo, dada la infraestructura de conectividad, el factor económico de los estudiantes y docentes no es posible hacer un uso efectivo de dichas plataformas educativas virtuales. De modo que, más bien se crea una saturación de las tecnologías, las cuales, no conlleva realmente a garantizar las condiciones idóneas para el aprendizaje (Mila, 2018). De tal manera que, el liderazgo del personal jerárquico y directivo debe identificar los nudos críticos y cuellos de botella de la institución educativa para luego elaborar propuestas de solución; sin embargo, en el Perú, solo el $20 \%$ de todas las empresas públicas y privadas realiza esfuerzos e inversiones para hacer seguimiento al crecimiento y cumplimiento de las tareas del líder (Salvador, 2017).

El problema es que se aprecia una realidad compleja e incierta donde estudiantes y docentes de la comunidad de aprendizaje víctorraulina manifiestan dificultades vinculadas al manejo de los procesos educativos mediados por las TIC, en un contexto de pandemia ocasionado por Covid-19, en el cual la educación virtual obtiene un papel prioritario y fundamental. En este contexto, se vuelve indispensable explorar nuevos modelos pedagógicos orientados hacia la virtualidad y, que permitan la interacción y la participación en una dimensión colaborativa y cooperativa. Debido a que, ha ocurrido una transición forzosa del modelo educativo basado en la presencialidad a otro sustentado a través de las TIC.

En tal sentido, se plantea la siguiente interrogante de investigación: ¿Cuál es la experiencia del personal jerárquico en relación al liderazgo mediado a través de las Tecnologías de Información y 
Comunicación en un contexto de trabajo remoto?

\subsection{EL LIDERAZGO}

Para Aldana (2019), el liderazgo se refiere a la capacidad de influencia que tieneun individuo sobre un grupo social, en función de conseguir apoyo en la obtención de los objetivos organizacionales. De esa manera, se consigue apoyoen la toma de decisión de la dirección. Todo ello, en función de lograr los objetivos organizacionales.

El liderazgo se consolida por medio de la influencia interpersonal entre los sujetos que constituyen una organización. Así,cada situación de la vida diaria se vuelve en una oportunidad para consolidar el proceso de comunicación y colaboración hacia el logro de los objetivos comunes.En tal sentido, el liderazgo constituye un rol fundamental para las organizaciones puesto que es necesario coordinar los grupos humanos en atención a una experiencia laboral satisfactoria para el grupo de involucrados, de forma tal quese sientan respaldados por una figura que ocupa un lugar superior y así alcanzarun mejor desempeño laboral.

Para Salvador (2017), el liderazgo está constituido bajo un enfoque de organización de aquellas acciones que se realizan en un colectivo en pro de la organización. De tal manera que, las diferentes expresiones humanas como la comunicación, la convivencia en comunidad y la cultura entre otros aspectos, sevan conjugando con una serie de políticas institucionales a fin de lograr la solidaridad y el trabajo en equipo en atención de las necesidades de la empresa.

\subsection{CARACTERÍSTICAS DE UN LÍDER}

Para Aldana (2019), el líder posee diversas características que lo vuelvenuna persona apreciada entre sus colaboradores, puesto que, comparten esos mismos principios y valores. A continuación, se describen algunas de ellas:

- Autoridad. El líder se convierte en un modelo a seguir a través del ejercicio de la autoridad y la influencia.

- Responsabilidad. El líder responsable aumenta el nivel de compromiso institucional de sus colaboradores.

- Decisión. El funcionamiento del equipo de trabajo depende en mayor porciónde decisiones acertadas de quien los dirige y éstas son determinantes en el cumplimiento de las metas organizacionales (Salvador, 2017).

- Comunicación. Los líderes deben caracterizarse por su capacidad de transmitirsus ideas hacia sus colaboradores y escucharlos activamente (Aldana, 2019). 
- Respeto. Los mejores lideres infunden respeto a través de la admiración de sus colaboradores y se consigue con un buen trato (Salvador, 2017).

- Visión. Es la capacidad del líder de ver más allá que permiten los ojos ordinarios, se trata de proyectarse hacia el futuro. El líder tiene la función decompartir su visión con el resto del equipo.

- Carisma. Es una característica que incide en la creación de una atmosfera detrabajo agradable. Esto se sustenta mediante el respeto y la comunicación asertiva (Aldana, 2019).

- Optimismo. El líder ejerce confianza entre sus colaboradores cuando le transmite fortaleza ante la adversidad. Esto, es importante para aumentar la motivación dentro del equipo de trabajo y conseguir mejores resultados (Salvador, 2017).

- Trabajo en equipo. Las individualidades deben hacerse a un lado, a fin de evitar afectar los intereses del colectivo.

\subsection{ESTILOS DEL LIDERAZGO}

El estilo de liderazgo, se constituye por el conjunto de características y rasgos personales que tiene el líder para gestionar grupos de personas. En este sentido, la influencia interpersonal determina el estilo que el líder ejerce al momento de coordinar, dirigir, guiar y motivar, entre otros factores. Desde luego, existen diversos estilos de liderazgo con marcadasdiferencias entre sí y, cada uno de ellos posee sus propias fortalezas y debilidades (Becerra \& Bermudez, 2020). A continuación, se describen algunos estilos de liderazgo:

- Liderazgo democrático: se apoya en un estilo participativo y tiene en consideración la perspectiva de todos los colaboradores. Este tipo de liderazgo delega funciones y promueve la toma de decisiones de manera coordinada (Aldana, 2019).

- Liderazgo apático: este tipo de líderes no asumen su responsabilidad y ceden sus obligaciones a sus subordinados. A este estilo, no le importa el cumplimiento de las actividades que realizan sus colaboradores. Poseen unbajo compromiso hacia la organización.

- Liderazgo transformacional y transaccional: bajo la dirección efectiva de esteestilo se consiguen mejores resultados para la organización; puesto que, toma en consideración aspectos como el apoyo socioemocional, el intercambio de experiencias, escucha activa y la comunicación asertiva, entre otros factores de inspiración (Nieto-Rivas, 2021).

\subsection{LIDERAZGO EDUCATIVO}

El liderazgo educativo se relaciona directamente con el rol que ejerce el director de una institución educativa en beneficio del estudiante, principalmente. Al respecto Araya-Guzmán et al. (2019), 
concuerdan con el concepto de que el liderazgo educativo llevado a cabo por el director de toda institución es clave enla consecución de mejoras en el aprendizaje de los estudiantes, mediante la gestión apropiada de recursos humanos y también materiales de la institución.

Cabe resaltar que hablar de liderazgo educativo, no solo hace referencia al nivel directriz, esto es al del director de la entidad educativa; también se da a nivel del aula con cada docente, motivando, ejerciendo su rol de líder en el aula,promoviendo la mejora de las actividades tendientes a lograr un excelente desempeño en cada estudiante. Igualmente es necesario mencionar que un estudiante puede ser líder en el aula, y es importante captarlos desde el inicio (Deng et al., 2019).

\subsection{LIDERAZGO ADAPTATIVO}

En la actualidad existen varios enfoques de liderazgo, uno que está llamando la atención es el denominado liderazgo adaptativo que según Gutierrez(2019), se fundamenta en el entendimiento de las relaciones entre las personas que conforman una institución o comunidad para lograr los objetivos prácticos propuestos de modo teórico, aplicando conceptos de la sociología predominantemente.

Es interesante la propuesta ya que resalta el aspecto del ser humano siempre cambiante, siempre voluble, dependiente de su contexto, por ello la relación con varias disciplinas entre ellas, la sociología, la ciencia política e incluso la biología evolucionista del ser humano (Condori, 2019).

\subsection{CLIMA INSTITUCIONAL}

El logro de los objetivos organizacionales tiene como factor determinantela influencia que el clima laboral tiene sobre el entorno en el que se desenvuelven los trabajadores, puesto que, en cualquier organización el talento humano es el recurso de mayor importancia. Así mismo, un adecuado clima laboral se gestiona en condiciones agradables, tanto para la organización, como para los trabajadores;situación que, en gran medida depende del estilo de liderazgo ejercido por los directivos de la empresa, según afirma Salvador (2017).

En este sentido, el óptimo funcionamiento de la organización se ve altamente influenciado por un equipo de trabajadores motivados y comprometidos con la organización consiguiendo como respuesta, por un lado, alta productividad; y por otro, satisfacción entre los empleados.

\subsection{DATOS ESTADÍSTICOS Y OTRA INFORMACIÓN RELACIONADA CON LA PROBLEMÁTICA ABORDADA}

La sociedad de la información y del conocimiento, emerge como un nuevo orden social, en que las TIC ocupan un rol preponderante. Sin embargo, no todoslos actores de la sociedad están preparados para 
asumir estos desafíos o no cuentan con la inversión necesaria para proyectos de infraestructura y alfabetización digital, lo que ha originado el aumento de las brechas digitales entre los países desarrollados y subdesarrollados, como las siguientes:

- Brecha intergeneracional: caracterizada por las diferencias en cuanto al manejo de las capacidades tecnológicas, principalmente las TIC entre los nativos digitales, es decir, los actores sociales que nacieron a comienzos delsiglo XXI, disfrutando del auge de dichas herramientas en su entorno natural.A diferencia de los migrantes digitales que están por encima de los 35 años de edad, a los cuales, les ha tocado realizar procesos de alfabetización digital, con el propósito de poder introducirse en el entorno de las TIC y los nuevos avances tecnológicos.

- Brecha digital de acceso: caracterizada por la ausencia o deficiente infraestructura básica que asegure la conexión a internet de manera eficiente.

- Brecha digital de uso: relacionada con la alfabetización digital, se refiere a la falta de competencias en materia de TIC, que impiden usar las tecnologíasde forma adecuada.

- Brecha digital de apropiación: caracterizada por la falta de empoderarse yde adaptar a los propios contextos el uso de las tecnologías, a fin de sacar elmayor provecho posible.

A continuación, se presentan algunos datos importantes del Instituto Nacionalde Estadística (INEI), que muestran una vista panorámica de la realidad de los hogares peruanos en relación al acceso a las TIC, de modo que, se puede deducir los aspectos subyacentes al fenómeno de las brechas digitales que están impactando el sistema educativo y, en consecuencia, la gestión efectiva del liderazgo directivo:

Tabla 1 Hogares con al menos un miembro que tiene teléfono celular (porcentajerespecto al total de hogares)

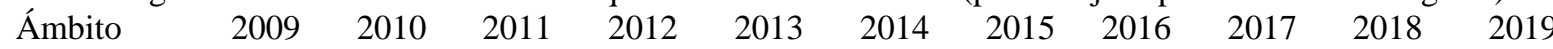
Geográfico

\begin{tabular}{cccccccccccc}
\hline $\begin{array}{c}\text { Lima } \\
\text { metropolitana }\end{array}$ & 80,3 & 83,3 & 85,6 & 88,8 & 88,5 & 91,1 & 92,9 & 93,3 & 94,0 & 95,1 & 96,3 \\
Sierra & 53,8 & 63,1 & 66,0 & 72,0 & 75,9 & 80,2 & 82,5 & 85,0 & 86,8 & 87,7 & 88,5 \\
\hline
\end{tabular}

Nota. INEI, 2020.

Tabla 2 Hogares con al menos un miembro que tiene una computadora (porcentajerespecto al total de hogares)

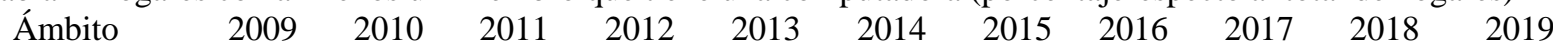

Geográfico

\begin{tabular}{cccccccccccc}
\hline $\begin{array}{c}\text { Lima } \\
\text { metropolitana }\end{array}$ & 34,7 & 36,0 & 40,7 & 48,3 & 49,8 & 51,7 & 49,7 & 51,1 & 51,7 & 52,8 & 47,3 \\
Sierra & 14,6 & 17,0 & 18,5 & 19,8 & 21,9 & 21,5 & 21,0 & 22,0 & 21,3 & 21,4 & 22,8 \\
\hline
\end{tabular}

Nota. INEI, 2020. 
Tabla 3 Hogares que acceden al servicio de internet (porcentaje respecto al total dehogares)

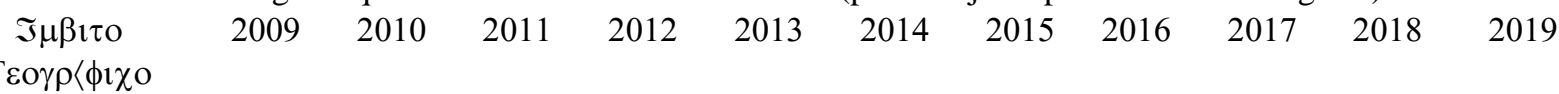

\begin{tabular}{|c|c|c|c|c|c|c|c|c|c|c|c|}
\hline $\begin{array}{c}\Lambda 1 \mu \alpha \\
\mu \varepsilon \tau \rho \circ \pi \mathrm{o} \lambda \iota \tau \alpha \nu \alpha\end{array}$ & 23,4 & 25,7 & 32,7 & 38,7 & 41,4 & 44,5 & 42,4 & 48,2 & 52,1 & 54,2 & 58,7 \\
\hline$\sum 1 \varepsilon \rho \rho \alpha$ & 4,5 & 5,6 & 6,9 & 8,8 & 9,7 & 10,0 & 10,5 & 12,0 & 12,9 & 13,7 & 18,9 \\
\hline
\end{tabular}

Nota. INEI, 2020.

En las tablas: 1, 2 y 3; se puede observar los hogares donde al menos unmiembro tiene un teléfono, una computadora y acceso a internet, respectivamente. Se ha realizado una comparación entre la región metropolitanade Lima, la cual, históricamente tiene mayor desarrollo de infraestructura tecnológica, calidad de vida y acceso a servicios básicos que las zonas de provincias del Perú. Se puede observar como en los tres aspectos anteriormentemencionados (acceso a celular, computadora e internet), durante los últimos 10 años (2009-2019), la región de la sierra tiene menor acceso a dichas tecnologías. De tal manera que, esto explica las dificultades que atraviesa el sistema educativo en la Sierra del Perú, donde precisamente se encuentra ubicado la unidad de análisis de este estudio (IESPP Víctor Raúl Haya de la Torre). Por tanto, no sorprende el aumento de las brechas digitales en esta región, quecada vez se viene acentuando, sobre todo en esta época de pandemia. En consecuencia, también, se ve perjudicado el ejercicio efectivo del liderazgo.

\section{MATERIAL Y MÉTODOS}

La presente investigación tuvo el siguiente desarrollo metodológico:

Diseño. El trabajo se condujo desde la perspectiva del enfoque cualitativo con un diseño fenomenológico hermenéutico para interpretar y entender las experiencias del personal directivo sobre el proceso de trabajo pedagógico remoto en contexto de pandemia ocasionado por el Covid-19. Asimismo, se profundizó el conocimiento del fenómeno objeto de estudio mediante un proceso de inmersión personal con la técnica de observación participante, así como también, a través de entrevistas a profundidad.

Participantes. La población de análisis está constituida por 3 docentes que ocupan cargos jerárquicos en la comunidad objeto de estudio ya mencionada. Los mismos, constituyen un grupo heterogéneo de 2 hombres y una mujer con edades que oscilan entre los 45 a 70 años. Los mismos, se sometieron a un proceso de codificación, análisis (redes semánticas, coocurrencias y categorías emergentes) para interpretar los hallazgos significativos del estudio a la luz del contexto desarrollado.

Instrumento. Se usaron diversas técnicas e instrumentos para la recopilación de datos. Por un lado, se empleó la técnica de la observación participativa, se utilizó un diario de campo y memos en el software ATLAS.ti, los cuales, permiten registrar la memoria metodológica de la investigación, para luego 
incorporarla al proceso de análisis discursivo de acuerdo a lo observación en relación a las experiencias de los docentes directivos. Por otro lado, se aplicó la técnica de las entrevistas a profundidad con una guía de 15 preguntas orientadoras.

Procedimiento. Primero, se llevó a cabo un proceso de revisión bibliográfica en artículos de revistas indexadas por la base de datos Scopus, Ebsco, etc, con la intención de analizar la información de los últimos 5 años. Posteriormente, se preparó una guía de entrevista de 15 preguntas. Seguidamente, se seleccionaron los sujetos a analizar bajo criterios de inclusión (docentes directivos pertenecientes al IESPP Víctor Raúl Haya de la Torre, región la Libertad) y exclusión (docentes con menos de 2 años ocupando cargos directivos), a todos se les solicitó el debido consentimiento informado. Mediante el empleo de Google Meet, se condujeron las entrevistas a profundidad, las cuales, duraron aproximadamente una hora; Dichas entrevistas se procesaron con el Software ATLAS.ti, en las que surgieron categorías emergentes. La información recopilada y analizada se relacionó a los referentes teóricos.

\section{RESULTADOS}

El análisis de coocurrencias de los códigos-documento obtenidos a través de las entrevistas a profundidad revelan los siguientes hallazgos significativos:

Tabla 4 Coocurrencias de los códigos-documentos

\begin{tabular}{lcccccc}
\hline \multicolumn{1}{c}{ Sub categorías } & Abs. $\begin{array}{l}\text { Relativo de } \\
\text { la fila }\end{array}$ & $\begin{array}{l}\text { Relativo } \\
\text { de la } \\
\text { columna }\end{array}$ & $\begin{array}{l}\text { Relativo de } \\
\text { la tabla }\end{array}$ & $\begin{array}{l}\text { Relativo } \\
\text { de la fila }\end{array}$ & $\begin{array}{l}\text { Relativo } \\
\text { de la tabla }\end{array}$ \\
\hline $\begin{array}{l}\text { Acompañamiento y } \\
\text { monitoreo } \\
\text { Gr=123; GS=14 }\end{array}$ & 123 & $100,00 \%$ & $35,76 \%$ & $35,76 \%$ & $100,00 \%$ & $35,76 \%$ \\
$\begin{array}{l}\text { Dirección efectiva } \\
\text { Gr=115; GS=11 }\end{array}$ & 115 & $100,00 \%$ & $33,43 \%$ & $33,43 \%$ & $100,00 \%$ & $33,43 \%$ \\
$\begin{array}{l}\text { Soporte emocional } \\
\text { Gr=106; GS=13 }\end{array}$ & 106 & $100,00 \%$ & $30,81 \%$ & $30,81 \%$ & $100,00 \%$ & $30,81 \%$ \\
\hline Totales & 344 & $100,00 \%$ & $100,00 \%$ & $100,00 \%$ & $100,00 \%$ & $100,00 \%$ \\
\hline
\end{tabular}

En relación a las categorías emergentes, se obtuvo como resultando el índice de emergencia siguiente (IE), el cual, determina el proceso reconstructivo de la investigación que devela los aspectos subyacentes en función de la triangulación. En la Figura 1, se describe el proceso de transferencia de la información recopilada de las entrevistas a profundidad de los tres directivos hacia las sub categorías a priori. 
Figura 1 Diagrama de Sankey de los códigos-documentos organizados en sub categorías

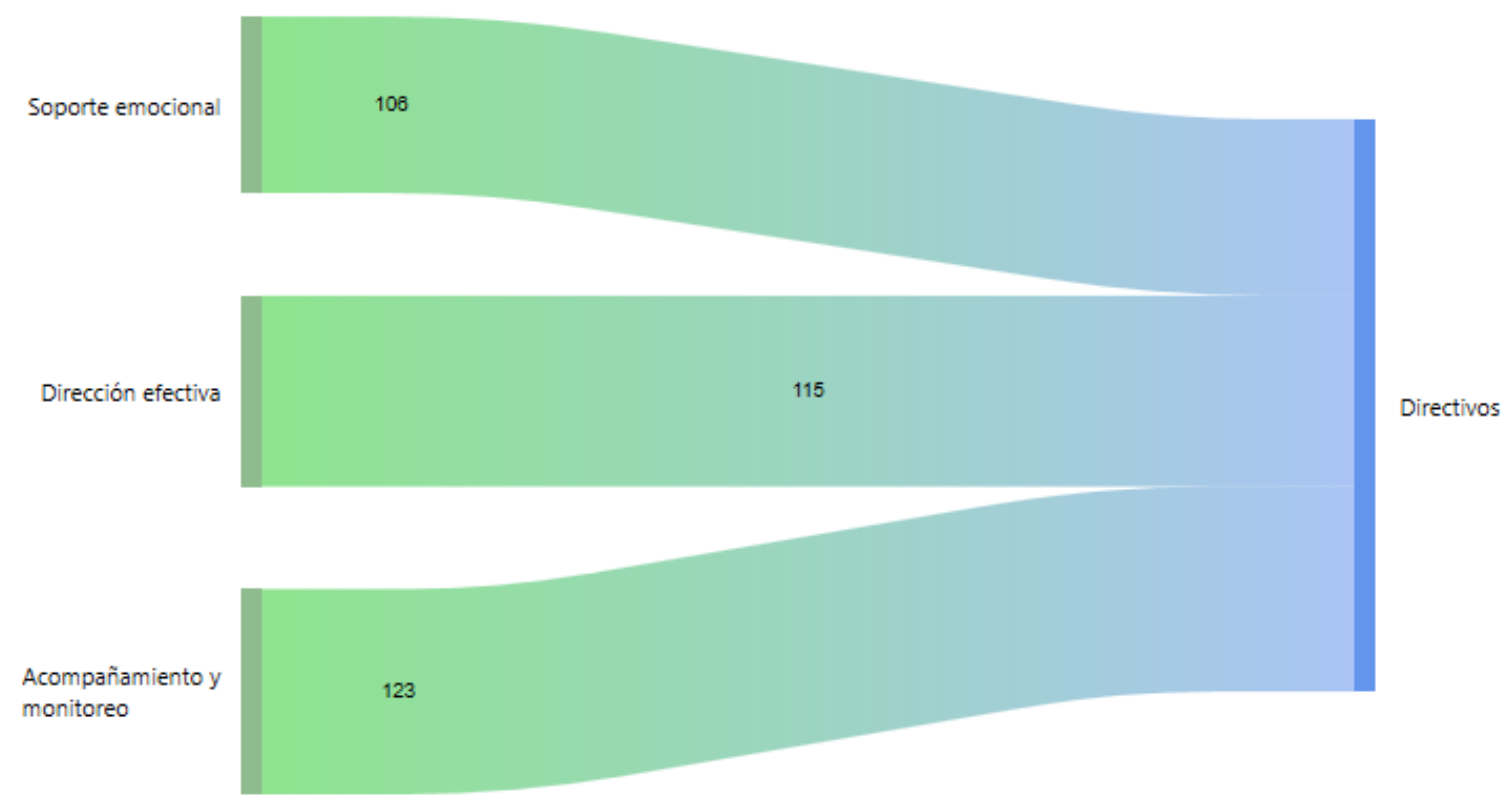

En relación a las categorías emergentes, mediante el proceso de reducción de datos se obtuvo como resultado el índice de emergencia (IE), de los 12 códigos más significativos de un total de 34 obtenidos originalmente.

A continuación, se muestra la Tabla 5, la cual, determina el proceso reconstructivo de la investigación que devela los aspectos subyacentes en función de la triangulación de datos a partir de diferentes fuentes de acopio de información.

Tabla 5 Resultados de los índices de emergencia (IE)

\begin{tabular}{llccc}
$\mathrm{N}^{\mathbf{o}}$ & Código & Enraizamiento & Densidad & $\begin{array}{c}\text { Índice de } \\
\text { Emergencia (IE) }\end{array}$ \\
\hline 1 & Empatía & 47 & 21 & 68 \\
2 & Comunicación asertiva & 41 & 9 & 50 \\
3 & Gestión oportuna & 36 & 13 & 49 \\
4 & Buen ánimo & 38 & 7 & 45 \\
5 & Trabajo colaborativo & 37 & 6 & 43 \\
6 & Intercambio de experiencias & 30 & 10 & 40 \\
7 & Delegación de funciones & 25 & 14 & 39 \\
8 & Escucha activa & 27 & 12 & 39 \\
9 & Adecuación de las TIC & 23 & 13 & 36 \\
10 & Soporte emocional & 26 & 9 & 35 \\
11 & Cumplimiento de tareas & 24 & 7 & 31 \\
12 & Dirección efectiva & 17 & 13 & 30 \\
\hline & Totales & 371 & 134 & 505 \\
\hline
\end{tabular}

Nota. Esta tabla permite apreciar la reducción de datos de los nuevos códigos que surgieron a posteriori, tomando como criterio los 12 códigos con el mayor índice de emergencia obtenido. 
Una vez finalizados los análisis de redes semánticas, coocurrencias y emergencias, se tiene la información suficiente para interpretar los hallazgos en el contexto en que se desarrollaron (relativización) de los datos. En tal sentido, es importante saber cuál es la experiencia del personal jerárquico en relación al liderazgo mediado a través de las Tecnologías de Información y Comunicación en un contexto de trabajo remoto. A tales efectos, se precisan a continuación, los resultados obtenidos en las tres sub categorías pertenecientes a la categoría 1: Experiencias del liderazgo.

\section{Sub categoría 1: Dirección efectiva}

Se encontró que la dirección efectiva está asociada con la gestión oportuna de las decisiones que se toman de manera consensuada. De modo que, cuando se presentan situaciones se debe analizar y reflexionar dicha problemática escuchando activamente a todos los actores involucrados de tal manera que, se produzca una convergencia de opiniones grupales. Además de ello, se debe enseñar con el ejemplo para que el personal docente sienta un trato cordial, con la intención de brindarle algún mensajito de aliento muy necesario en medio de esta coyuntura.

Por otro lado, se evidenció que la dirección efectiva del liderazgo se sustenta en los procesos de delegación de funciones con el propósito de ir generando autonomía; de modo que, los docentes puedan tener la confianza de actuar y de comunicar las diversas situaciones del día a día; desde luego, todo ello, en base al respeto que nunca se debe perder porque el manejo de las emociones es muy importante y no se debe descuidar.

A continuación, en la Figura 2, se aprecia la red semántica de la construcción hermenéutica de la sub categoría: Dirección efectiva. 
Figura 2 Red semántica de la sub categoría 1: Dirección efectiva

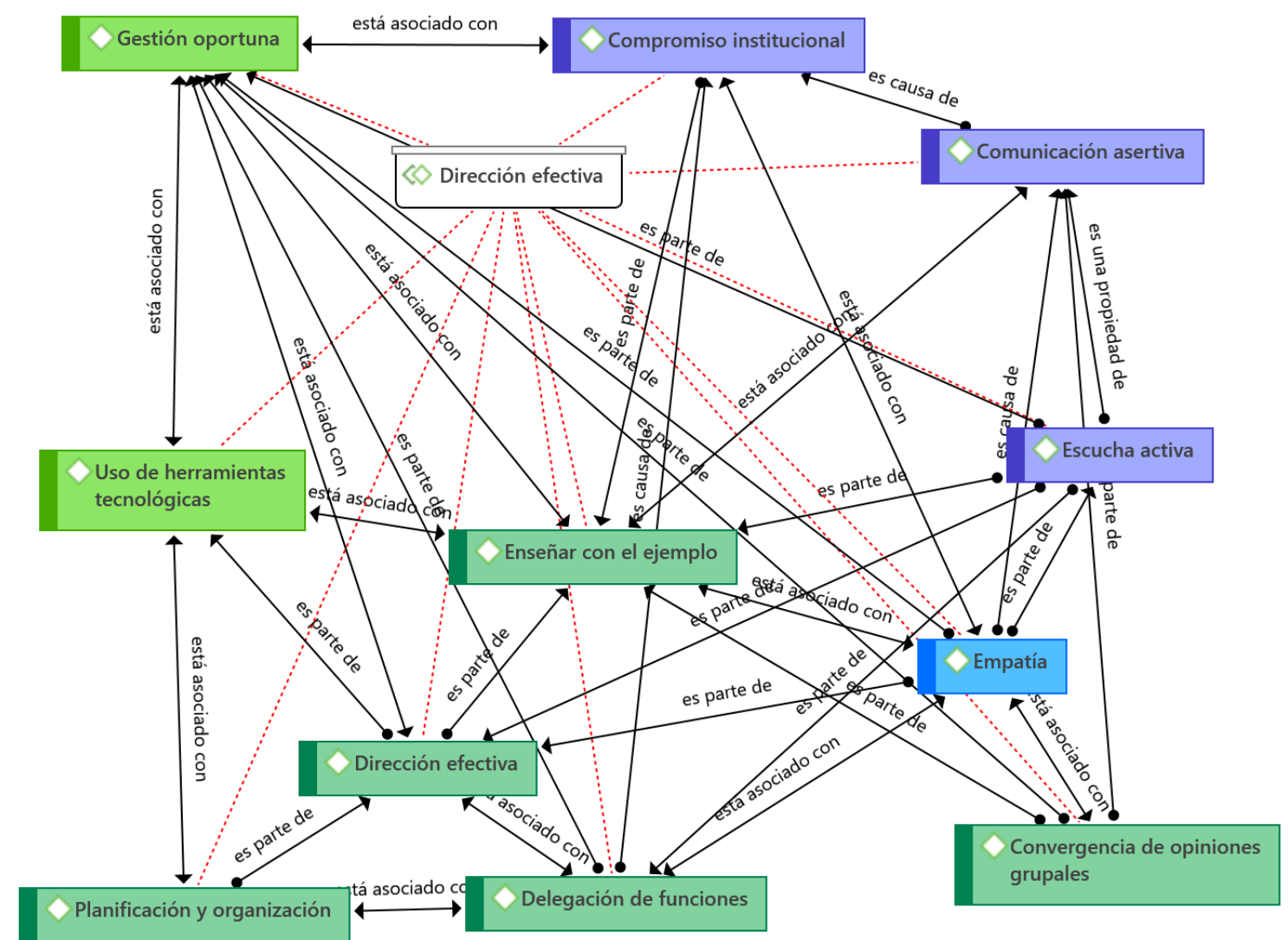

Nota. Esta figura permite apreciar las relaciones de significado entre los distintos códigos que intervienen en la sub categoría: Dirección efectiva

Sub categoría 2: Acompañamiento y monitoreo

Se encontró que para llevar a cabo el acompañamiento y monitoreo en primera instancia los directivos deben ser empáticos con el resto del equipo, maestros, padres de familia, entre otros. Puesto que, en estos tiempos de pandemia cualquiera puede padecer afectaciones de salud que le impiden ejercer su trabajo; por ello, el liderazgo de los directivos es útil para brindarles soporte emocional; sobre todo escuchando activamente las sugerencias y recomendaciones del personal acerca de lo que hay que hacer, en función de entender la situación con una mirada panorámica. Esto también contribuye a fomentar los procesos de participación y toma de la iniciativa. A continuación, en la Figura 3, se aprecia la red semántica de la construcción hermenéutica de la sub categoría: Acompañamiento y monitoreo. 
Figura 3 Red semántica de la sub categoría 2: Acompañamiento y monitoreo

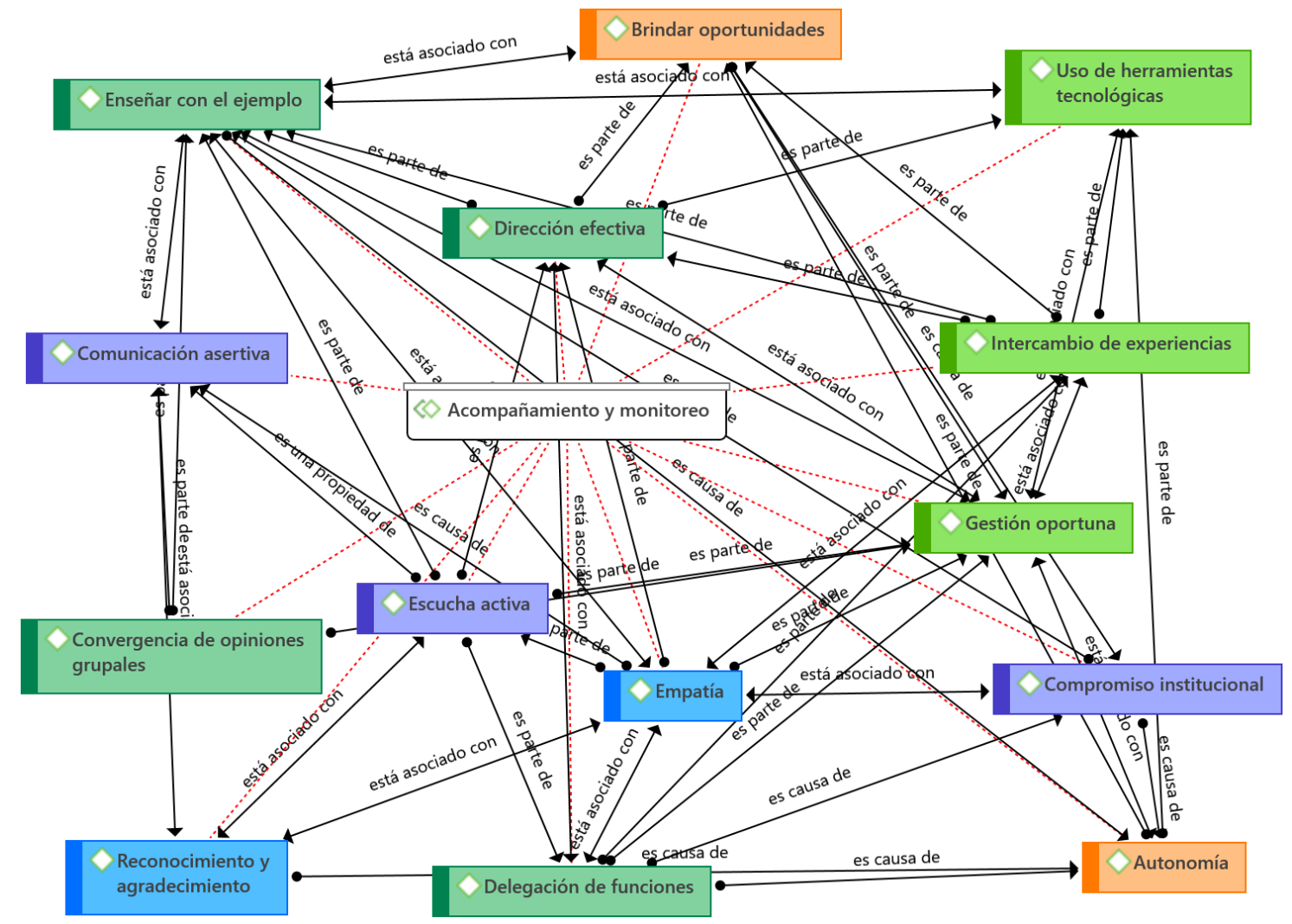

Nota. Esta figura permite apreciar las relaciones de significado entre los distintos códigos que intervienen en la sub categoría: Acompañamiento y monitoreo

Sub categoría 3: Soporte emocional

Se encontró que la solidaridad es uno de los valores más practicados como herramienta de liderazgo efectivo en tiempo de pandemia; puesto que todos se necesitan entre sí, todos se dan la mano para apoyarse en las distintas funciones que se llevan a cabo en las instituciones educativas. Antes de todo es importante ver cómo es la persona y también ver sus sentimientos, ser empáticos.

Con respecto al valor del sentido de responsabilidad, se ha encontrado que la mayoría del personal docente cumple con sus funciones; a pesar de todo, se encuentran altamente motivados dado el sentido de vocación que tienen por la carrera magisterial. De igual modo, el liderazgo de los directivos se centra en reconocer y agradecer de manera pública y privada el trabajo de los maestros; sobre todo el proceso arduo que llevan para adecuarse al uso de las TIC y así, brindar sus sesiones de aprendizaje en un contexto de trabajo pedagógico remoto. A continuación, en la Figura 4, se aprecia la red semántica de la construcción hermenéutica de la sub categoría: Soporte emocional. 
Figura 4 Red semántica de la sub categoría 3: Soporte emocional

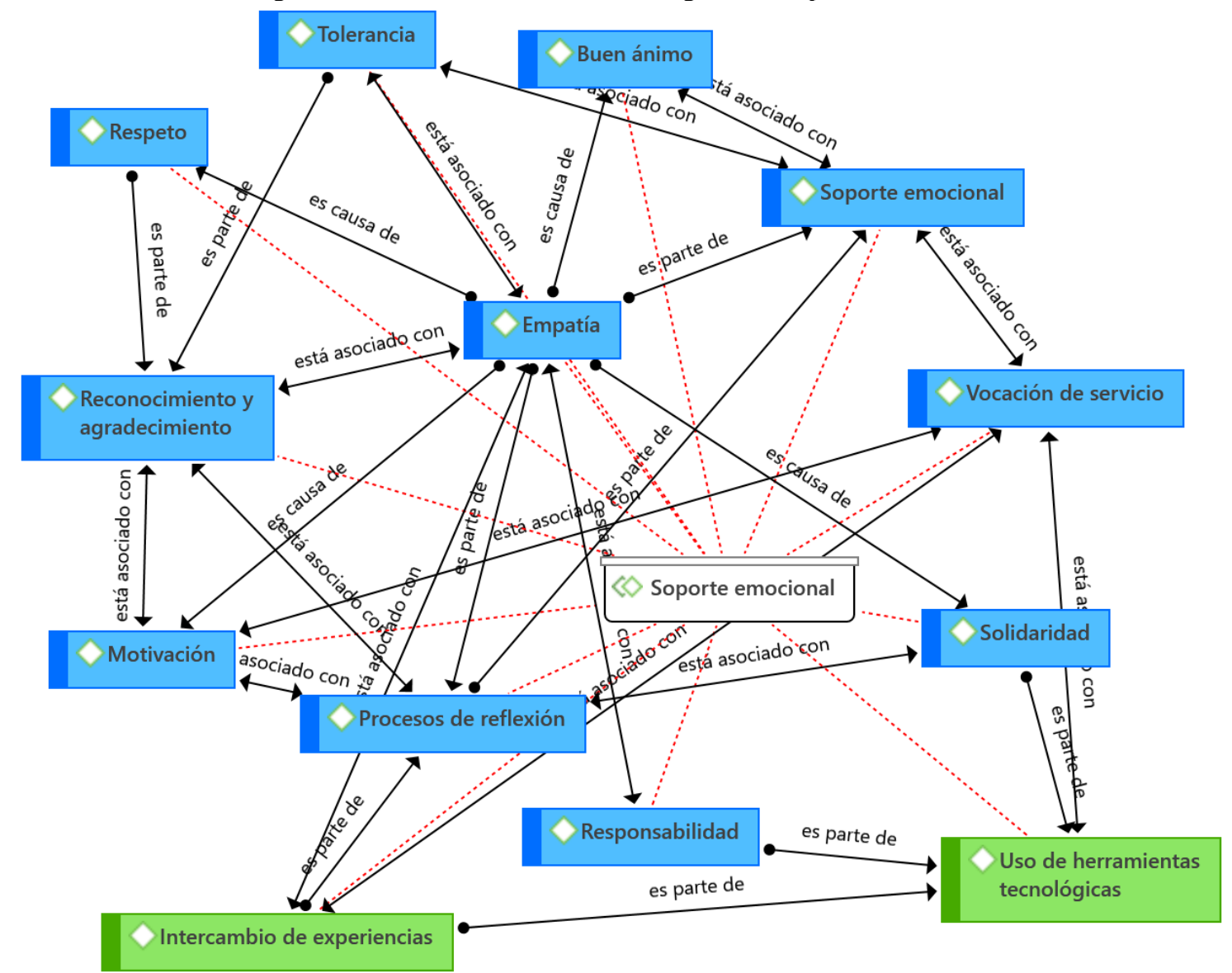

Nota. Esta figura permite apreciar las relaciones de significado entre los distintos códigos que intervienen en la sub categoría: Soporte emocional.

A continuación, se puede observar en la Figura 5, los grupos de códigos que brotan como sub categorías emergentes resultados del índice de emergencia (IE), evidenciado en la Tabla 5, a fin de dar nuevas luces acerca de las experiencias del liderazgo directivo con respecto al trabajo pedagógico en contexto de pandemia. 
Figura 5 Diagrama de Sankey de los códigos emergentes

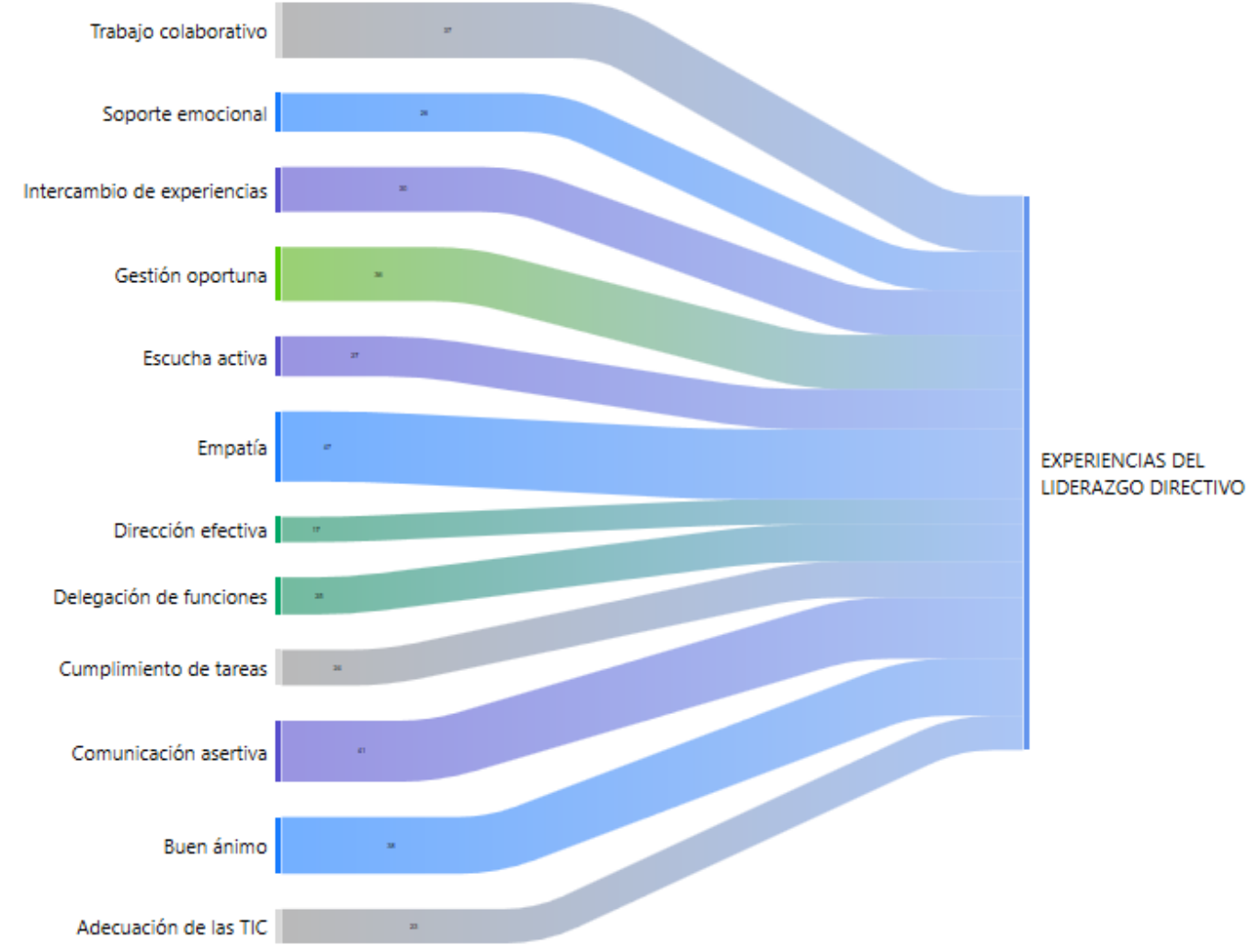

Figura 6 Red semántica de la categoría 1: Experiencias del liderazgo

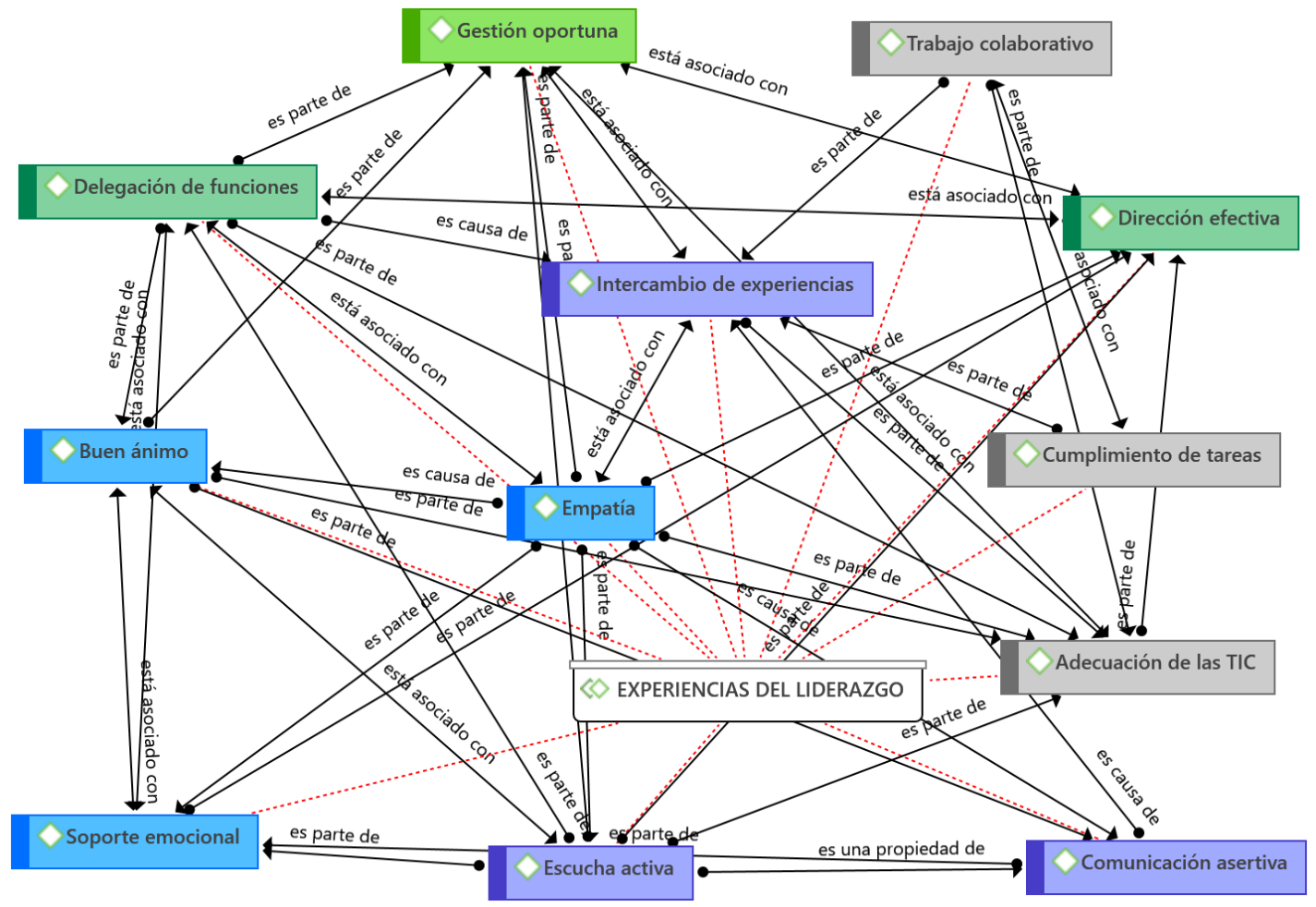

Nota. Esta figura permite apreciar las relaciones de significado entre los distintos códigos emergentes, ya reducidos que intervienen en la categoría 1: Experiencias del liderazgo. 


\section{DISCUSIÓN Y CONCLUSIONES}

El rol del líder, el director, en una institución educativa, independientementede su estilo de liderazgo, es de fundamental importancia en la consecución de los objetivos institucionales de mejora de la calidad de la enseñanza y aprendizaje de los estudiantes, en tiempos de pandemia, especialmente cuando la influencia de las dificultades tecnológicas es bastante apreciable, tanto del lado docente como del estudiante, debido al trabajo en forma remota, altamente dependiente de las tecnologías de información y comunicaciones. Por ello, es esencial un adecuado acompañamiento y monitoreo que reconozca y agradezca el trabajo realizado a efectos de generar empatía y elevar el compromiso institucional, así como también, consolide la convergencia de las opiniones grupales. Esto concuerda con lo hallado en el estudio de Amador (2017), quien asegura que un buen líder debe congregar y lograr una convivencia armoniosa en la comunidad educativa.

La promoción de valores de la institución educativa, tomando como base, el comportamiento de la plana directiva, puede propiciar un ambiente amigable dondela colaboración entre pares prospere, en beneficio de la gestión académica y administrativa de la institución, y por ende del personal directivo y docentes quienesson los beneficiarios directos, así como de los estudiantes y personal administrativo, como beneficiarios indirectos. En tal sentido, es esencial promover soporte emocional y manejo de las nuevas herramientas tecnológicas aplicadas a la docencia mediante un plan de capacitación en materia de liderazgo y en el uso de tecnologías de información y comunicaciones que involucre a la plana directiva y a los docentes. De modo que, se puedan fortalecer las habilidades de los miembros de la institución educativa; sin descuidar aspectos esenciales como la solidaridad, vocación de servicio, el respeto, la tolerancia y el buen ánimo; los cuales, son vitales para encarar los desafíos que supone la educación virtual. Esto, concuerda con lo encontrado en el trabajo de Aldana (2019), quien afirma que el líder debe desarrollar actitudes de fraternidad entre sus colaboradores, puesto que, es necesario compartir y enseñar los principios y valores organizacionales. 


\section{REFERENCIAS BIBLIOGRÁFICAS}

Aldana Chávez, B. R. (2019). Liderazgo y Clima Laboral en la empresa “Cleaning Corp.” Universidad Central del Ecuador.

Amador, C. (2017) Ventajas del liderazgo educativo en educación superior. Revista Iberoamericana para la Investigación y el Desarrollo Educativo, 8(15),14-26.

http://www.scielo.org.mx/scielo.php?script=sci_arttext\&pid=S2007- 74672017000200817

Araya-Guzmán, S., Jiménez, C. S., Toledo, E. E. G., Bío-bío, U., Ramírez Correa, P., \& Alfaro-Pérez, J. (2019). Explorando la relación entre Gestión del Conocimiento y Rendimiento Organizativo en Organizaciones Educativas. Revista Ibérica de Sistemas e Tecnologias de Informação, 17(June), 12.

Becerra, C. V., \& Bermudez, J. J. (2020). El clima laboral en la biblioteca universitaria: el caso de seis bibliotecas universitarias en Colombia. Investigación Bibliotecológica: Archivonomía, Bibliotecología e Información, 34(84), 59. https://doi.org/10.22201/iibi.24488321xe.2020.84.58165

Betemi, V. (2019) Organizaciones escolares ante un mundo cambiante, Neuro gestión, 14(4), 34-48. https://webdelmaestrocmf.com/portal/victor-rawlins-neurogestion- escolarorganizaciones-escolaresinteligentes-ante-un-mundo-cambiante/ Boada, I. E. (2017). Liderazgo y Clima Laboral en CLINMEYD S.A. Northospital. Universidad Central del Ecuador.

Bucheli, M. G., \& Bastidas Terán, F. A. (2020). WhatsApp como recurso para el trabajo grupal en $\begin{array}{llll}\text { estudiantes } & \text { universitarios. } & \text { Apertura, } & \text { 72(2), }\end{array}$ https://doi.org/http://dx.doi.org/10.32870/Ap.v12n2.1911

Condori, Y. (2019). Liderazgo y gestión del conocimiento de los directivos del Instituto Superior Pedagógico Público de Puno. Revista Innova Educación, 1(2), 189-196. https://doi.org/10.35622/j.rie.2019.02.005

Deng, W., Li, X., Wu, H., \& Xu, G. (2019). Student leadership and academic performance. China Economic Review. https://doi.org/10.1016/j.chieco.2019.101389

Gutierrez, Y. (2019). El liderazgo adaptativo de Ronald Heifetz. Características, alcances y limitaciones. Universidad del Rosario. http://repository.urosario.edu.co/handle/10336/19871

INEI. (2020). Instituto Nacional de Estadística e Informática INEI. https://www.inei.gob.pe/estadisticas/indice-tematico/brecha-digital-de-genero-7920/

Kochen, G. (2020). La gestión directiva o el liderazgo educativo en tiempos de pandemia. Innovaciones Educativas, 22(33), 9-14. https://doi.org/10.22458/ie.v22i33.3349

Lescano, L. (2017). Cómo fortalecer el clima y la cultura de servicio a través del liderazgo de servicio del mando intermedio: caso de estudio en una compañía multinacional. Revista Empresa y Humanismo, XX(1), 65-96. https://doi.org/10.15581/015.xx.1.65-96

Mila, E. C. (2018). La motivación por la lectura y su papel en el logro de la comprensión lectura. Propuesta de investigación pedagógica. Revista Dilemas Contemporáneos: Educación, Política y Valores, 6(1), 129.

Nieto-Rivas, E. A. (2021). Transformación de la acción comunicativa en comunidades de aprendizaje. 
HELIOS, 5(1), 103-124. https://doi.org/http://doi.org/10.22497/Helios.51.5102

Salvador, R. (2017). Liderazgo y clima laboral de los colaboradores de una empresa dedicada a la cobranza en Lima, 2016. Universidad César Vallejo. 